\title{
ISOCHIROTHERIUM sp: PISTA DE UM GIGANTESCO TECODONTE NA FORMAC̣ÃO ANTENOR NAVARRO (TRIÁSSICO), SOUSA, PARAIBBA, BRASIL
}

\author{
GIUSEPPE LEONARDI*
}

\begin{abstract}
A bipedal chirotherian trackway, from the Antenor Navarro formation (Sousa, Paraiba) is here described, and classified as Isochiroterium $\mathrm{sp.}$

The discovery made it possible to date the formation às Triassic, most probably Middle to Upper Triassic.
\end{abstract}

INTRODUÇÃo As pistas de tetrápodes encontradas até agora no nordeste brasileiro eram exclusivamente dinossaurianas, sendo representadas as subordens Coelurosauria e Carnosauria e, na subordem Ornithopoda, as famílias Hypsilophodontidae e Iguanodontidae (Moraes, 1924; Huene, 1931; Leonardi, $1978 a-b$, $1979 a-b)$.

Em expediçáo de julho de 1977 à Bacia do Rio do Peixe, Paraíba, além do mais foi descoberto na localidade "Serrote do Letreiro", município de Sousa, 10,5 km NW desta cidade, na estrada para Uiraúna, na margem setentrional da bacia, um interessante conjunto de pistas e, entre elas, uma quiroteriana.

As pistas encontram-se impressas em camadas assentadas poucos metros acima da base do pacote sedimentar da bacia, apoiando discordantemente sobre os gnaisses do embasamento pré-câmbrico; são constituídas por arenitos arcoseanos grosseiros com camadas conglomeráticas, todos fortemente litificados, por vezes com aspecto de quartzitos.

Trata-se da formaça Antenor Navarro (W. D. Costa, fide J.P.T. Albuquerque, 1971), cuja idade, bas tante incerta até agora, será definida mais abaixo; sua posição estratigráfica dentro do grupo Rio do Peixe é claramente inferior em relação à formação Sousa (W.D. Costa, fide J.P.T. Albuquerque, 1971) e esta está subjacente d̀ formação Piranhas (J.P.T. Albuquerque, 1971).

Nas baixas colinas do "Serrote do Letreiro", quase todas as superficies rochosas deixadas a descoberto pelo solo e pela vegetação espinhosa da caatinga apresenta, juntamente as incisðes rupestres (chamadas popularmente "letreiro") dos índios Cariri, pistas de tetrápodes que se podem dividir em quatro grupos:

a) grandes pegadas de má qualidade de forma geral arredondada, de dinossauros bípedes de porte médio mas de corporatura pesada (segundo resulta da "onda" circular de lama sublevada ao redor da pegada);

b) pegadas isoladas e pistas de dinossauros Theropoda (sigla: SOSL 4 e 5, Fig. 1), de porte pequeno e médio atribuídos (Leonardi 1978b) a uma forma pequena de Eubrontes E. Hitchcock, 1845, ou a uma forma médio-grande de Grallator E. Hitchcock, 1858, com artelho III curto e passo duplo breve; a incerteza entre os dois gêneros depende do fato de essas formas carecerem de uma boa revisão;

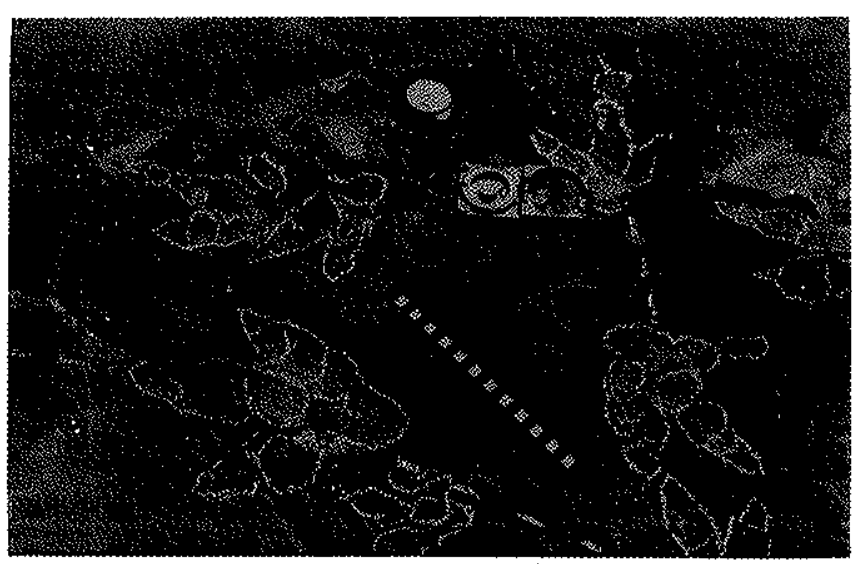

Figura 1 - Rastros de Terópodos associados à pista de Isochirotherium nas superfícies rochosas do "Serrote do Letreiro", Sousa, Paraiba; formação Antenor Navarro

c) uma pequena pegada avulsa de tipo muito esquisito, constituída por quatro almofadinhas de forma poligonal que podemos comparar, por enquanto, com as mão de algumas formas de Batrachopus, por exemplo $B$. obesus, ou também com as pegadas dinossauróides incompletas ilustradas por Bronner, G. e Demathieu, G. (1977) (sigla: SOSL 3); e

d) uma pista evidentemente quiroteriana (SOSL 2), que é objeto do presente artigo, com três pegadas: a primeira bem conservada e as outras úteis apenas para indicar os elementos fundamentais da pista.

A PISTA QUIROTERIANA - DESCRIÇÃo Pista de três pegadas consecutivas de animal bípede de grande porte (Figs. 2 e 4); passo duplo, $185 \mathrm{~cm}$; passo oblíquo, $96 \mathrm{~cm}$; ângulo do passo, $165^{\circ}$; largura externa da pista, $40 \mathrm{~cm}$; vão da pista, $\sim 7 \mathrm{~cm}$; bitola das patas, $20,5 \mathrm{~cm}$; comprimento médio da pegada, $40,3 \mathrm{~cm}$; lar. gura média da pegada, $24 \mathrm{~cm}$; comprimento médio da planta, $16,5 \mathrm{~cm}$; e largura média da planta, $19,7 \mathrm{~cm}$.

$A$ angulaçáo do eixo da pegada com o eixo da pista é difícil de se calcular pela forma e disposiçâo dos dedos; na primeira pegada, é positiva $\left(16^{\circ}\right)$ e, nas outras, era provavelmente próxima a zero. Não resulta nenhuma impressão das mãos ou da cauda.

* Conselho Nacional de Desenvolvimento Científico e Tecnológico (CNPq) - Rua Xavier da Silva, 227, CEP 84100, Ponta Grossa, PR 


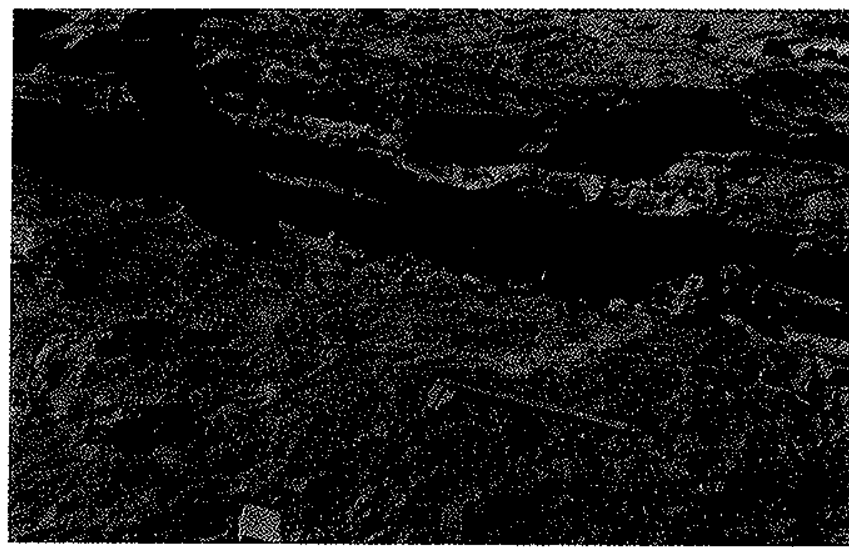

Figura 2 - Camadas de arenito grosseiro da formação Antenor Navarro, com uma rara pista de Isochirotherium. Mesma localidade da Fig. 1

A pegada do pé é subplantígrada, pentadátila, assimétrica, mesaxônica mas com tendência à paraxonia no que diz respeito aos dedos I-IV; dedo I sempre separado dos demais; dedos II-IV pouco divergentes e quase apertados entre si, com as bases dos dedos III e IV, e aparentemente também do $V$ na primeira pegada, reunidas por uma única calosidade correspondente à fusão das respectivas almofadinhas falangeal-metatarsais; dedo I relativamente curto; dedo II longo quase como o III, que é o mais longo de todos; IV menor que o II mas maior que o I.

Os dedos I-IV săo no conjunto compridos e finos, dirigidos inicialmente para fora em relação ao eixo da pista, mas depois de novo para dentro. Almofadinhas digitais evidentes, sem calosidades, garras muito fortes mas um tanto obtusas; particularmente grande parece ser a do dedo II.

$\mathrm{O}$ dedo $\mathrm{V}$, em forma de apoio lateral, está em forte abdução (ângulo IV-V $>100^{\circ}$ ); é provido de uma forte

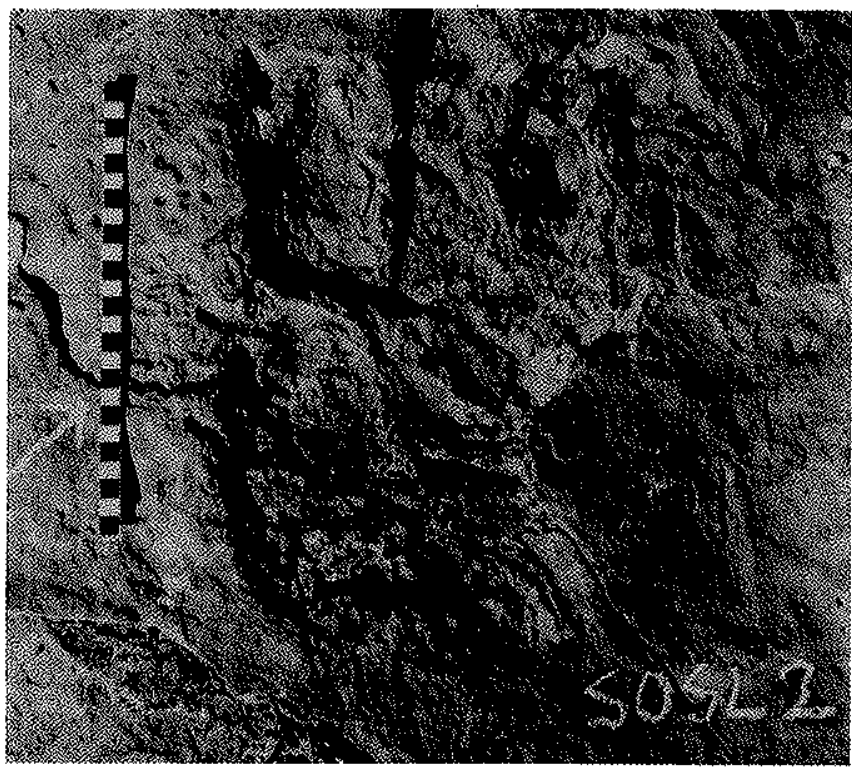

Figura 3 - A primeira pegada da pista de Isochirotherium. Reparar no comprimento quase igual dos dedos II e III

almofada falangeal-metatarsal, não completamente dividida da linha das almofadinhas dos dedos I-IV nem de uma pequena planta. $O$ animal, gigantesco em seu gênero e muito pesado, apoiava-se sobre uma superfície relativamente grande.

$\mathrm{O}$ índice passo oblíquo:comprimento da pegada é baixo $(2,307)$ em relação, por exemplo, a espécimes de Synaptichnium priscum DEMATHIEU, 1967 ( 3); Isochirotherium soergeli HAUBOLD, 1967 ( 3,1); Chirotherium sickleri KAUP, $1835 b(\sim 4,35)$; ou, enfim, em relação a Brachychirotherium harrarense HAUBOLD, 1967 (5). O indice passo duplo:comprimento da pegada é também, obviamente, baixo $(4,54)$ (Synaptichnium, 5,3; Isochirotherium, 6,2; Chirotherium, 8,4; e Brachychirotherium, 9,4). Em alguns espécimes de Iso-

Tabela 1 - Mediç̧̌es dos dedos só na primeira pegada $(\mathrm{em} \mathrm{cm})$

\begin{tabular}{lrrrrrr}
\hline & I & II & III & IV & V \\
\hline Comprimento do dedo & 19,5 & $\sim 26,0$ & 26,5 & $\sim 20,0$ & $-1,5$ \\
Comprimento do dedo livre & 15,0 & 19,5 & $\sim 20,7$ & $\sim 16,0$ & 8,5 \\
Comprimento da porção falangeal & 17,0 & $\sim 22,7$ & 23,5 & - & - \\
Largura máxima do dedo & 5,4 & 5,5 & 5,3 & $\sim 4,0$ & 4,75 \\
\hline
\end{tabular}

Tabela 2

\begin{tabular}{lccc}
\hline & Primeira pegada & Segunda pegada & Terceira pegada \\
\hline Ângulo interdigital I-II & $18^{\circ}$ & $7^{\circ}$ & $16^{\circ}$ \\
Ângulo interdigital II-III & $8^{\circ}$ & - & $3^{\circ}$ \\
Ângulo interdigital III-IV & $4^{\circ}$ & - & - \\
Ângulo interdigital IV-V & $121^{\circ}$ & - & - \\
Ângulo interdigital II-IV & $12^{\circ}$ & - & - \\
Ângulo interdigital I-V & $149^{\circ}$ & & \\
Ângulo da cruz & $83^{\circ}$ & & \\
\hline
\end{tabular}


chiroiherium hessbergensis HAUBOLD, 1970a, os dois valores saio muito parecidos aos nossos $(2,32$ e 4,52), assim também no tipo de lsochirotherium felenci COU. REL e DEMATHIEU, $1976(2,35$ e 4,8).

Provavelmente, devido ao bipedismo habitual ou eventual, o animal precisava firmar mais seus pés com um passo relativamente curto; também o ângulo do pas. so nåo é particularmente alto.

DISCUSSÃo Năo há dúvida de que se trata de uma pista quiroteriana. $O$ fato de um quiroteroide ser bipede não é caso único. Lembramos: Chirotherium bipedale ABEL, 1935 (segundo Haubold, 1971 sinônimo de Isochirotherium hercu/is), pista de má qualidade do Keuper inglês; a pista bipede de duas pegadas, do Moenkopi, ilustrada por Peabody (1948, págs. 393-4 e estampa 45), provavelmente un Isochirotherium; e ainda a pista bipede, tambem de Isochirotherium sp. do Ladiniano ou Carniano da Suísa, registrada por $G$. Bronner e G. Demathieu (1977).

Por outro lado, de um ponto de vista geral, a subordem Pseudosuchia Zittel, 1887-1890, à qual é atribuída comumente a maioria das pistas quiroterianas, é caracterizada pela presença de tendências ao bipedismo; e é possivel qua mesmo pseudo-suquianos habitualmente quadrúpedes se levantassem em posição bípede na corrida rápida.

- Nâo parece que a pista em estudo faça necessária a instituicão de um novo gênero, năo apresentando no estilo geral características profundamente diferentes.

Podemos excluir logo, entre os quatro gêneros quiroterianos, Synaptichnium NOPCSA, 1923, além do mais pela ausência em nossa forma de estrutura geral lacertóide com predominância do IV dedo, e pela notável abdução do dedo V. Também podemos excluir Brachychiroterium BEURLEN, 1950, pelas seguintes características que não há em nossa pista: almofadinhas digitais redondas procedentes da fusảo de várias almofadinhas originárias; unhas pequenas; ausência quase completa da porção falangeal do dedo $V$; e dedos geralmente um tanto curtos. Chirotherium KAUP, 1835, e Isochirotherium Haubold, 1971, apresentam caracteristicas nas quais nossa forma pode ser parcialmente reconhecida. Além do mais, ambos os gêneros abrangem formas gigantes; o segundo abrange formas bípedes; Chirotherium barthii já foi encontrado na América do Sul (Higueras, Las Heras, Província de Mendoza, Argentina; Rusconi, 1952; Peabody, 1955).

Parece que nossa forma se encaixa mais na descricão típica de Isochirotherium pelo comprimento quase igual dos dedos II e III, com o III só levemente predominante; base do $V$ transversal-proximal em relação aos dedos II-IV; estes constituem um grupo muito apertado e saem de uma única calosidade falangeal-metatarsal, como em algumas formas de Isochirotherium.

Sem dúvida haveria os elementos para se fazer uma nova espécie de Isochirotherium, o que faremos oportunamente; lembraremos os valores dos ângulos interdigitais, a curvatura dos dedos para dentro, a extrema ab- duçăo do V, a separação do I em relaçăo ao grupo IIIV, o bipedismo, o baixo ângulo do passo, etc. Pretende-se, porém, por um lado, controlar, com posteriores buscas no canpo', se este tipo de pista é sempre bípede; e, de outro lado, ficar fiéis ao princípio de instituir novos taxa apenas quando os tipos forem recolhidos em um museu ou instituição que garantisse a conservação. Estamos estudando, com a diretoria e o Departamento de Paleontologia do Museu Nacional do Rio de Janeiro, um projeto concreto de corte e transporte para aquela instituição desta e de outras pistas do nordeste e centrooeste brasileiro.

Limitar-nos-emos, por enquanto, a classificar a pista do "Serrote do Letreiro" (Sousa, Paraíba) como Isochirotherium $\mathrm{sp}$.

DATAÇÃO E CORRELAÇÃO A descoberta da pista em esiudo é importante porque é a primeira pista quiroteriana encontrada no Brasil e a segunda publicada na América Latina; o primeiro sinal da presença de Tecodontes na América do Sul ao norte do Rio Grande do Sul; e, além do mais, trata-se de um dos maiores espécimes, talvez o maior, de Chirotheriidae.

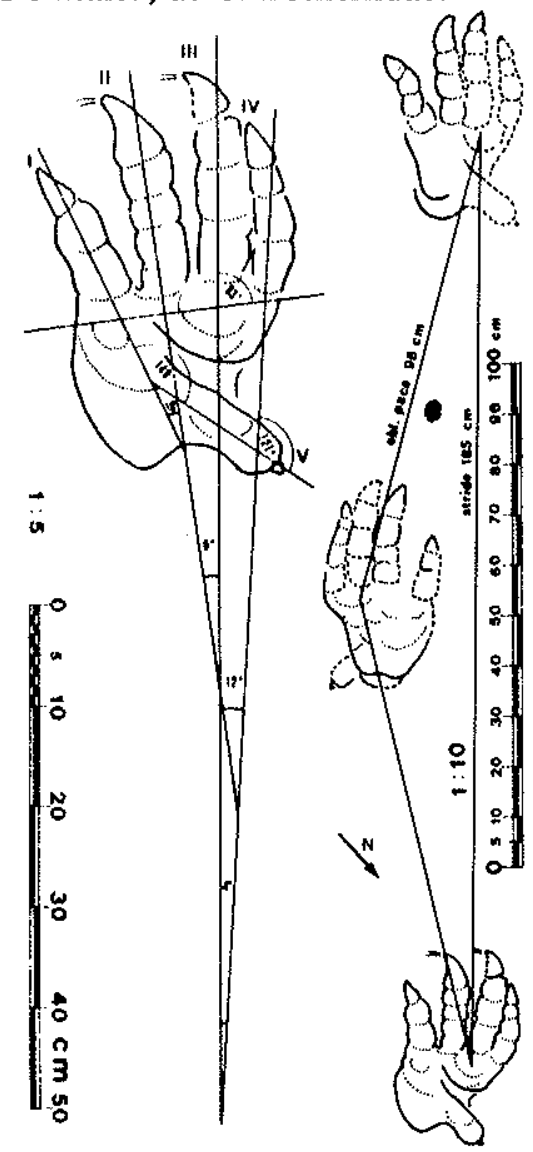

Figura 4 - Desenho da pista de Isochirotherium e da primeira pegada em escala maior, com indicação dos métodos de medj. ção. A linha continua representa o contorno conservado; a pontilhada, as dobras da pele e as margens das almofadinhas; e a linha interrompida indica as margens duvidosas ou reconstruídas

${ }^{1}$ Em recente expedição à localidade referida (dezembro de 1979), encontramos mais três pegadas avulsas, de má qualidade , atribuíveis à mesma forma. 
Um dos aspectos mais interessantes do achado é a possibilidade de datar e correlacionar a formaça Antenor Navarro e o grupo Rio do Peixe em geral, e ter um elemento útil de comparação para a datação de outras formaçð̌es continentais nordestinas.

Os quiroterianos são típicos do Triássico². Esta morfofamilia, extremamente difundida e muito bem conhecida e estudada nos continentes boreais, mais rara por enquanto nos outros continentes, é comumente atribuída a Tecodontes de várias subordens, principalmente Pseudosuchia e Aetosauria'; estes são registrados, a nivel osteologico, exclusivamente no Triássico.

Podemos então, desde já, atribuir a formação Antenor Navarro ao Triássico enquanto o inteiro pacote sedimentar do Rio do Peixe foi considerado cretáceo (geralmente neocomiano) por Crandall (1910), Moraes (1924), Huene (1931), Beurlen (1964), Braun (1969), Mabesoone e Campanha (1974) e Brito (1976) e jurássico por Anjos et al. (1967). Albuquerque (1971) julga correlata com a formação Tacaratu (Mauriti), portanto devoniana, a formação A. Navarro e jurássica as formaçðes Sousa e Piranhas.

O gênero Isochirotherium ao qual atribuimos a pista em estudo é conhecido até agora em terrenos do Triássico inferior e médio, nos continentes boreais; por outro lado, as pistas associadas foram classificadas preliminarmente (Leonardi, 1978b) como Eubronies E. HITCHCOCK, 1845, ou Grallator E. HITCHCOCK, 1858, ambos atribuídos a Theropoda; e ambas săo for. mas conhecidas em terrenos do Triássico superior ao Jurássico inferior. O primeiro gênero foi encontrado até agora $\mathrm{em}$ terrenos do Ladiniano (mais comumente do Carniano) ao Liássico superior (Europa, América do Norte, África central e meridional, e Austrália) e, o segundo, do Carniano ao Infraliássico (Europa, América do Norte e Sul da África).

Os dados icnológicos indicariam portanto, como datação mais provável, o Triássico médio ou superior, com particular ênfase para o Ladiniano e o Carniano; precisa-se levar em conta, porém, as limitaçðes do método, sobretudo em um continente no qual a Icnologia de vertebrados (e muitas vezes a própria Paleontologia de vertebrados) só apresenta dados raros e esparsos.

Sendo que as três formaçð̃es do grupo Rio do Peixe têm entre si passagem gradual e constituem um único ciclo sedimentar, o abaixamento para o Triássico da formação inferior provavelmente acarreta também o abaixamento das duas outras.
ATRIBUiÇÃo Dependendo dessa datação, as grandes pegadas referidas acima sob o item $a$ poderiam eventualmente pertencer a Prosaurópodos; a pegada do item $c$ poderia ser efetivamente a impressão da mâ de um protossuquiano ou mais provavelmente, devido ao tamanho, do pé de um dinossauro não especificado, se. gundo resultou também de critérios morfológicos; e as pistas do item $b$ pertenceriam a coelurossauros de dimensðes avantajadas ou a um carnossauro médio-pequeno.

Finalmente, o réptil responsável pela pista quiroteriana poderia ser procurado, pela idade e pelo tamantio, entre os Rauisuchidae que alcançam, no proprio território brasileiro, na formação Santa Maria, tamambo gigantesco, bem proporcionado ao das pegadas agui ilus. tradas.

Os rauissuquianos, porém, são conhecidos como quadrúpedes (embora alguns deles pudessem eventual. mente exercer bipedismos facultativo) e a estrutura dos pés năo corresponde à anatomia indicada pelas pezadas, particularmente o Il radius é bern mais curto que colil e mais curto que oIV.

O organismo das pistas há de ser procurado, eni todo o caso, entre os PSEUDOSUCHIA s.s. e mais precisamente eni um grupo que estava evoluindo do quadrupedismo a um estágio de bipedismo lento, lerdo e facul. tativo.

Agradecimentos Um especial agradecimente pós. tumo ao mestre e amigo Dr. L.h. Price, do DNLM do Rio de Janeiro, recentemente desaparecido; suá rica biblioteca particular generosamente colocada a men total dispor, suas críticas e seus conselhos e sua propris pre. sença no pais foram para mim um grande inceritio a pesquisa no campo da Paleo-herpetologia.

Desejo agradecer cordialmente ao Prof. Dr. C. Demathieu pela interessante discussão sobre a pista guiroteriana ( 7 de fevereiro de 1978).

Amigáveis agradecimentos ao Dr. J.F. Bonanarte, do Museu Argentino de Ciencias Naturales (Buenos Aires), e ao Geol. D.A. Campos, chefe da seçấo d: Paleontologia do DNPM (Rio de Janeiro), pelas valiosas informaçðes, opiniðes e pelo fornecimento de material bibliográfico.

Finalmente, destaco com gratidðo a generosa colaboração logística da Prefeitura de Sousa (PB) e, especialmente, dos prefeitos pro tempore senhores Clarence Pires de Sá e Sinval Gonçalves Ribeiro.

2 O único caso, até agora, de Chirotherium com certeza não-triássico é o dos "Arenitos de Val Gardena", do Turingiano superior (Conti et al., 1977).

${ }^{3}$ Considera-se aqui a ordem Thecodontia dividida em quatro subordens: Proterosuchia, Pseudosuchia (incluindo a família Rauisuchidae), Aetosauria e Phytosauria. 


\section{BIBLIOGRAFIA}

ALBUQUERQUE, J.P.T. de - 1971 - Inventário Hidrogeológico do Nordeste. Folha n? 15, Jaguaribe, SE. SUDENE. Hidrogeologia, Recife, 32, $187 \mathrm{pp}$. BAIRD, D. - 1979 - Current work. Ichnology Newsletter 10: 20.

BRAUN, O.P.G. - 1969 - Geologia da Bacia do Rio do Peixe, Nordeste do Brasil. DNPM, 4\% distr. NE: 1-23.

BRITO, I.A.M. - 1976 - Geologia das Bacias sedimentares brasileiras. Col. trab. apres. I.II.III Semanas Est. Geol, U.F.R.R.J. Itagual, Rio de Janeiro: 53-73.

BRONNER, G, e DEMATHIEU, G. - 1977 - Premières traces de Reptiles archosauriens dans le Trias autochtone des Aiguilles Rouges (Col des Corbeaux, Vieil Emosson, Valais Suisse). Constequences paleogégraphiques et chronostratigraphiques. C. R. Acad. Sc. Paris 285: 649-652.

CAMPOS, M. de, et al. - 1974 - Projeto Rio Jaguaribe; Relatório de fotointerpretacto. CPRM, Recife: 32-35.

CHARIG, A.J., et al. -1970 - The classification of the Proterosuchia. Biol. Jour. of the Linnean Soc. 2: 125-171.

CONTI, M.A., LEONARDI, G., MARIOTTI, N. e NICOSIA, U. - 1977 Tetrapod footprints of the "Val Gardena Sandstone" (North Italy). Their paleontological, stratigraphic and paleoenvironmental meaning. Palaeontographia Italica 70 (N.S.40): 1-91.

COUREL, L. e DEMATHIEU, G. - 1963 - Les empreintes de pas fossiles de la bordure Est e Nord-Est du Massif Central. Bull. Scient, de Bourgogne 20 (1961-62): 73-92.

COUREL, L. e DEMATHIEU, G. - 1976 - Une Ichnofaune reptilienne remarcable dans les grès Triasiques de Largentière (Ardèche, France). Palaeontographica, A, 151: 194-216.

COUREL, L., DEMATHIEU, G. e BUFFARD, R. - 1968 - Empreintes de pas de vertebrés et stratigraphie du Trias. Bull. Soc. Geol. France, VII S., 10: 275-281.

DEMATHIEU, G. - 1970 - Les empreintes de pas de vertébrés du Trias de la bordure nord-est du Massif Central. Paris, Cahiers de Paltont. C.N.R.S., Paris, $211 \mathrm{pp}$.

DEMATHIEU, G. e HAUBOLD, H. - 1972 - Stratigraphische Tetrapodenfahrten aus der terrestrischen Trias Europas. Geologie 21(7): 802-836.

DEMATHIEU, G. e HAUBOLD, H. - 1974 - Evolution und Lebensgemeinschaft terrestrischer Tetrapoden nach ihren Fahrten in der Trias. Freiberger Forsch..H. - C 298 - Paldont.: 51-72.

GINSEURG, L.. LAPPARENT, A.F. de e TAQUET, Ph. -1968 - Piste de Chirotherium dans le Trias du Niger. C. R. Acad. Sc. Paris 266: 2056-2058.

HAUBOLD, H. -1969 - Die Evolution der Archosaurier in der Trias aus der Sicht ihrer Fahrten, Hercynia 6(1): 90-106.

HAUBOLD, H. - 1971 - Ichnia Amphibiorum et Reptiliorum fossilium. In:
KUHN, O., Handbuch der Palaohtrpetologie., Stuttgart, G. Fischer, v, 18, ix $+124 \mathrm{pp}$.

HAUBOLD, H. $-1971 \mathrm{~b}-$. Die Tetrapodenfahrten des Buntsandsteins in der Deutschen Demokratischen Republik und in Westdeutschland und ihre Aequivalente in der gesamten Trias. Paltont. Abh, A 4(3): 395-548.

HAUBOLD, H. - 1974 - Die fossilen Saurierfahrten. Wittenberg Lutherstadt, A. Ziemsen, 168 pp.

HUENE, F.F. von - 1931 - Verschiedene mesozoische Wirbeltierreste aus SOdamerika. N. Jb. Min. etc. 66(B): 181-198.

LEONARDI, G. - 1978a - Ornithischian trackways of the Corda Formation (Jurassic), Goiás, Brazil. Acta. I Congr. Latinoam. Paleont. Buenos Aires, no prelo.

LEONARDI, G, - 1978b - Dez novas pistas de Dinossauros (THEROPODA MARSH, 1881) na bacia do Rio do Peixe, Paraiba, Brasil. Acta 1 Congr. Latinoam. Paleont., Buenos Aires, no prelo.

LEONARDI, G. - 1979a - Nota preliminar sobre seis pistas de dinossauros Ornithischia da Bacia do Rio do Peixe (Cretáceo inferior) em Sousa, Paraiba, Brasil. An. Acad. brasil. Cienc. 51(3): 501-516.

LEONARDI, G. -1979 b - New Archosaurian Trackways from the Rio do Peixe Basin, Paralba, Brazil. Ann. Univ. Ferrara (N.S.), S.IX, 5(14): 239-246. LEONARDI, P. - 1959 - Ome Chiroteriane Triassiche Spagnole. Est. Geol. Inst. "Lucas Mallada" 15(4I-44): 235-245.

LULL, R.S. 1942 - Triassic footprints from Argentina. Amer. Jour. Sci. 240: $421-425$.

MABESOONE, J.M. e CAMPANHA, V.A. - 1974 - Sinopse de Estratigrafia das sequetencias sedimentares do Nordeste Brasileiro. Bol. Núcleo NE Soc. brasil. Geol. 2: 7-22.

MORAES, L.J. - 1924 - Serras e montanhas do Nordeste. Brasil. Insp. Obr. contra Seccas, Série l-D Publ. 58, 2 v., xi $+122+122$ pp.

PEABODY, F.E. - 1948 - Reptile and Amphybian Trackways from the Lowe Triassic Moenkopi formation of Arizona and Utah. Bull. Dept. Geol. Sci. 27(8): $295-468$.

PEABODY, F.E. - 1955 - Occurrence of Chirotherium in South America. Bull. Geol. Soc. Amer. 66: 239-240.

ROMER, A.S. - 1972 - The Chatiares (Argentina) Triassic Reptile Fauna. XVI. Thecodont Classification. Breviora, Mus. Comp. Zool. 395: 1-24.

RUSCONI, C. - 1952 - Rastros de Patas de Reptiles Permicos de Mendoza. Rev. Hist, y Geogr. de Cuyo 3(3): 1-14.

SOERGEL, W. - 1925 - Die Fahrten der Chirotheria. Eine palkontologische Studie. Jena, G. Fischer. 92 pp.

Recebido em 11 de junho de 1980. 